UCRL-ID-119854

\title{
Heavy Ion Physics at the Brookhaven Alternating Gradient Synchrotron
}

\author{
T. C. Sangster
}

January 30, 1995

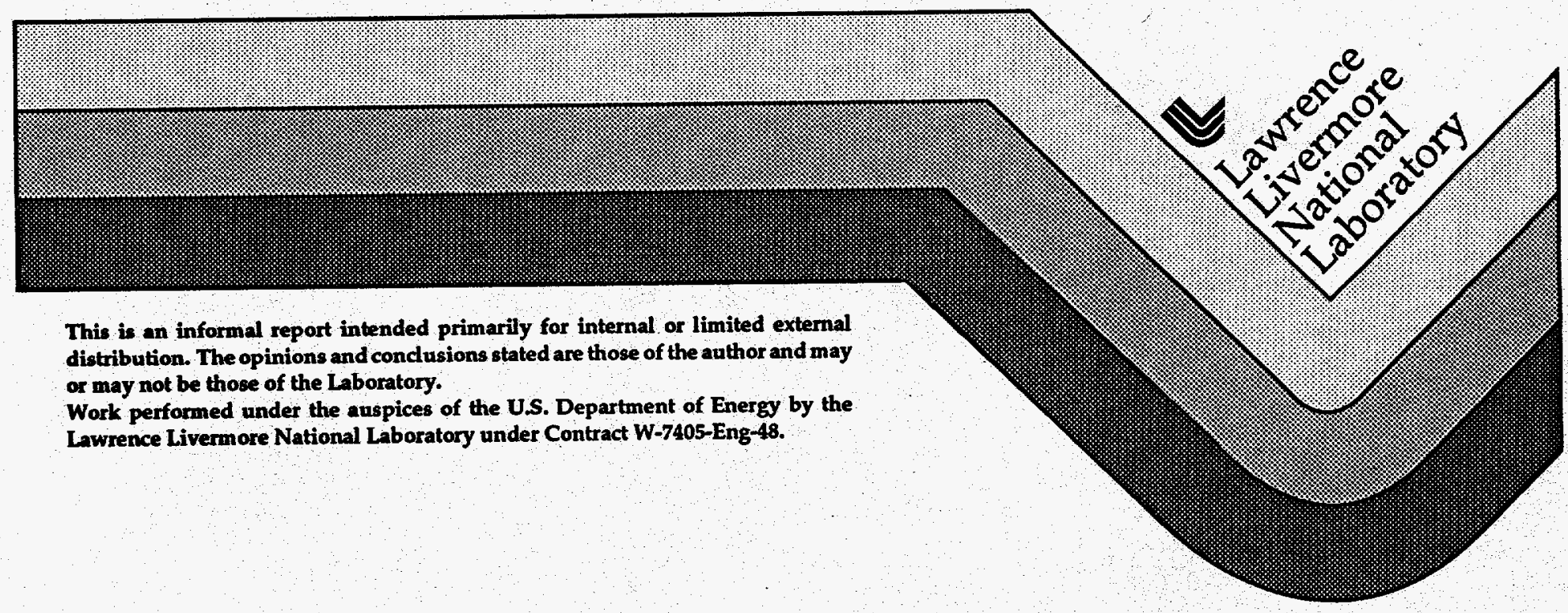




\section{DISCLAIMER}

This document was prepared as an account of work sponsored by an agency of the United States Government. Neither the United States Government nor the University of California nor any of their employees, makes any warranty, express or implied, or assumes any legal liability or responsibility for the accuracy, completeness, or usefulness of any information, apparatus, product, or process disclosed, or represents that its use would not infringe privately owned rights. Reference herein to any specific commercial products, process, or service by trade name, trademark, manufacturer, or otherwise, does not necessarily constitute or imply its endorsement, recommendation, or favoring by the United States Government or the University of California. The views and opinions of authors expressed herein do not necessarily state or reflect those of the United States Government or the University of California, and shall not be used for advertising or product endorsement purposes.

This report has been reproduced

directly from the best available copy.

Available to DOE and DOE contractors from the Office of Scientific and Technical Information

P.0. Box 62, Oak Ridge, TN 37831

Prices available from (615) 576-8401, FTS 626-8401

Available to the public from the

National Technical Information Service

U.S. Department of Commerce

5285 Port Royal Rd.

Springfield, VA 22161 


\section{DISCLAIMER}

Portions of this document may be illegible in electronic image products. Images are produced from the best available original document. 


\title{
Heavy Ion Physics at the Brookhaven Alternating Gradient Synchrotron
}

\author{
A Final Report for the Office of Scientific and Technical Information \\ on LDRD Project 92-DE-088 \\ Submitted by: \\ T. C. Sangster \\ Lawrence Livermore National Laboratory
}

\begin{abstract}
The collision of large nuclei at relativisitic energies is the only known mechanism for creating and studying the properties (equation-of-state, EOS) of extremely dense nuclear matter. At sufficiently high matter densities, one of the most exciting possibilities is the formation of a Quark Gluon Plasma (QGP). However, it is an extremely difficult task to determine the ultimate density achieved during these collisions due to the fleeting nature of the high density state (the lifetime of these states is typically a few times $10^{-24} \mathrm{~ns}$ ). We must rely on detailed comparisons between experimental measurements and complex Monte Carlo simulations of the colliding nuclei in order to extract meaningful estimates of the nuclear matter EOS. Our approach has been to study the behavior of the spectator matter (those protons and neutrons which do not directly interact during the collision) to determine the dynamics of the high density state which must necessarily influence the spectator matter as it decays. This report summarizes some of the key results of our study.
\end{abstract}




\section{Introduction}

The experimental work summarized in this report was performed at the Brookhaven National Laboratory Alternating Gradient Synchrotron as part of the E802 Collaboration. The specific experiment numbers are E859 (for the $14.6 \mathrm{GeV} / \mathrm{c} / \mathrm{A} \mathrm{Si}+\mathrm{A}$ reaction studies) and E866 (for the $11.7 \mathrm{GeV} / \mathrm{c} / \mathrm{A} \mathrm{Au}+\mathrm{Au}$ reaction studies). We proposed to measure pion, proton, deuteron and triton emission in relativisitic $\mathrm{Si}+\mathrm{A}$ and $\mathrm{Au}+\mathrm{Au}$ reactions at large laboratory angles correlated with collision impact parameter. Particle emission beyond the free scattering kinematic limit is a sensitive probe of rescattering mechanisms. Rescattering of the primary projectile nucleons and the secondary produced particles leads to the rapid increase in baryon density in the overlap region during the collision of the two nuclei. The pressure gradient across the collision zone further leads to collective phenomena such as hydrodynamical flow and shadowing. The collective response of the nuclear matter and produced particles are then a sensitive measure of the nuclear equation-ofstate. Therefore, we further proposed in experiment E866 to measure the reaction plane of the colliding nuclei on an event-by-event basis to directly study the collective flow and shadowing in relativistic $\mathrm{Au}+\mathrm{Au}$ collisions.

To perform these measurements, the LLNL Relativistic Heavy Ion Group constructed three detector subsystems for the basic E859 and E866 apparatus. The E859 Target Phoswich Array is described in [1]. For experiment E866, this array was completely rebuilt to increase the segmentation for the higher particle multiplicities expected with the Au beam reactions. The E866 Phoswich Array consists of 100 modules each capable of particle identification $(\pi, p, d, t)$ and kinetic energy measurement. In addition, the readout electronics for the E866 Array were upgraded and simplified from the NIMbased readout scheme in E859. The solid angle coverage of both arrays was nearly identical. The data from the two different detectors shown in Figure 1 compares remarkably well for the same target-projectile system.

We also designed and fabricated a high rate, high multiplicity tracking chamber for the primary E866 spectrometer. This chamber significantly enhanced the data collection capabilities of the spectrometer by extending the 
range of allowable track multiplicities thereby increasing the rapidity coverage of the spectrometer for the $\mathrm{Au}+\mathrm{Au}$ reactions.

Finally, to address the questions of collective phenomena in $\mathrm{Au}+\mathrm{Au}$ collisions, we designed and fabricated a Projectile Hodoscope. The hodoscope consists of two orthoganal scintillator slat arrays approximately 11 meters dowstream of the target. The rather simple conceptual idea is that the Hodoscope measures the deflection of the projectile spectator matter caused by the pressure gradient in the high density participant zone (the region of overlap between the two colliding nuclei). This deflection is quite small and the hodoscope segmentation is fairly high: $391-\mathrm{cm}$ wide by $40-\mathrm{cm}$ long $X$ slats and an equal number of identical $Y$ slats. Each slat is readout using two photomultiplier tubes and both pulse height and time-of-flight information are recorded for the reaction plane analysis (see Figure 2). To determine the reaction plane, we search for the $X$ and $Y$ charge centroid weighted additionally by the transverse momentum. Since we continuously monitor the position of the beam spot on the array (see Figure 3) as a function of time within the beam spill, the reaction plane is simply the plane defined by the line joining the beam spot and the charge centroid and the line of the beam. The milestone of the final year of this proposal was to install and commission the Projectile Hodoscope. This data has not yet appeared in any publication, however preliminary data is shown in the following section.

\section{Results}

Much of our work and the results are summarized in the publications listed at the end of this report [1-11]. Indeed, the final results of both the Si beam experiment, E859, and the initial experimental run of the $A u$ beam experiment, E866, including the detailed comparisons with the microscopic models RQMD and ARC, are presented in [11]. Detailed descriptions of these models can be found in the references of [11].

The final year of this project was devoted to the commissioning of the Projectile Hodoscope. Figures 2 through 4 show some of the preliminary Hodoscope data as well as our preliminary correlations with the protons measured in the Target Phoswich Array. The data in Figure 4 show the mean 
proton transverse momenta measured in the Phoswich Array projected into the reaction plane measured by the Hodoscope. The net negative momentum is the first indication for hydrodynamical flow in relativistic heavy ion collisions. These are important new data which will have a profound influence on our understanding of the reaction dynamics and nuclear equation-of-state. In addition, the observation of this signal indicates the utility of the reaction plane measured on an event-by-event basis. We expect that additional reaction plane correlations involving the produced particles will give us invaluable information on in-medium scattering cross sections, identical particle Hanbury-Brown-Twiss correlations and spectator shadowing.

\section{Summary}

We have made measurements which are crucial for our understanding of the nuclear equation-of-state. Working with microscopic collision models to make detailed comparisons with our data, we have demonstrated that it is possible to extract information related to the nuclear matter equation-of-state. The detector subsystems now in operation in experiment E866 at the Brookhaven Alternating Gradient Synchrotron will continue to provide unique information during future experiments. The Projectile Hodoscope, in particular, has proven to be an invaluable tool whose potential for new physics is only just now being realized. 


\section{References}

1. "A Phoswich Array for Relativistic Heavy Ion Collisions", J. B. Costales, H. C. Britt, M. N. Namboodiri, T. C. Sangster, J. H. Thomas and H. E. Wegner, Nucl. Instrum. and Meth. A330, 183 (1993); UCRL-JC-112259.

2. "Target Rapidity Baryon Distributions in $\mathrm{Si}+\mathrm{Au}$ and $\mathrm{Au}+\mathrm{Au}$ Collisions at 14.6A and 11.7A GeV/c", T. C. Sangster, J. B. Costales and M. N. Namboodiri, Proceedings of the Conference on Heavy Ion Physics at the AGS, MIT, Cambridge, MA, January 13-15, 1993; MITLNS-2158; UCRL-JC-112925.

3. "Target Rapidity Proton Distributions for Si+A Collisions at the AGS", Y. Akiba etal., Nucl Phys. A544, 445c (1992).

4. "Particle Production at High $\mathrm{P}_{\mathrm{t}}$ in $\mathrm{Si}+\mathrm{A}$ Collisions at $14.6 \mathrm{~A} \mathrm{GeV} / \mathrm{c}$ ", $\mathrm{Y}$. Akiba etal., Nucl. Phys. A544, 553c (1992).

5. "Recent Results from E802 and E859", T. Abbott etal., Nucl. Phys. A544, 237c (1992).

6. "Bose-Einstein Correlations of Kaons in $\mathrm{Si}+\mathrm{Au}$ Collisions at $14.6 \mathrm{~A} \mathrm{GeV} / \mathrm{c}^{\text {", }}$ Y. Akiba etal., Phys. Rev. Lett. 70, 1057 (1993).

7. "Global Transverse Energy Distributions in $\mathrm{Si}+\mathrm{Al}, \mathrm{Au}$ at $14.6 \mathrm{~A} \mathrm{GeV} / \mathrm{c}$ and $\mathrm{Au}+\mathrm{Au}$ at 11.6A GeV/c", L. Ahle etal., Phys. Lett. B332, 258 (1994).

8. " Recent Results from E859 using Si Beams at 14.6A GeV/c", Y. Akiba etal., Nucl. Phys. A566, 269c (1994).

9. "Backward Emission of Protons in Au+Au Collisions at $11.7 \mathrm{~A} \mathrm{GeV} / \mathrm{C}$ ", L. Ahle etal., Nucl. Phys. A566, 443c (1994).

10. "Experimental Status of the AGS Relativistic Heavy Ion Program", T. C. Sangster, Proceedings of the 27th International Conference on High Energy Physics, Glasgow, Scotland, July 20-27, 1994; UCRL-JC-118789.

11. "Baryon Emision at Target Rapidities", Y. Akiba etal., to be published in Phys. Rev. C; UCRL-JC-119319. 


\section{Figure Captions}

Figure 1: A comparison of the proton yields as a function of pseudorapidity for the $11.7 \mathrm{~A} \mathrm{GeV} / \mathrm{c} \mathrm{Au+Au}$ reaction taken with the E859 Target Phoswich Array and the new E866 Array. The two Arrays were designed to make measurements over the same kinematic region. The agreement demonstrates the consistency of our measurements between the two experiments, E859 and E866.

Figure 2: (Top) The geometric mean of the two photomultiplier tube (PMT) pulse heights for $X$ slat 14 showing the remarkable charge resolution of a single slat. (Bottom) The time-of-flight sum and difference spectra for the two PMTs. The width of the time sum distribution indicates a time resolution of approximately 225-ps. The width of the time difference spectrum corresponds directly to the length of the hodoscope slat: $48 \mathrm{ch} \times 25$-ps/ch $=1.2 \mathrm{~ns}$ times 30 $\mathrm{cm} / \mathrm{ns}$ (speed of light) equals $37-\mathrm{cm}$ (the length of the slat is $40-\mathrm{cm}$ ).

Figure 3: In all six panels, we show the slat position of of the beam (vertical axis) versus the spill time, an arbitrary measure of the time during the beam spill. Note that the location of the beam spot depends on the time during the spill. We must know the location of the beam very accurately to get the correct magnitude for the collective flow.

Figure 4: The preliminary mean transverse momentum of the protons measured in the Target Phoswich Array and projected into the Hodoscope reaction plane as a function of pseudorapidity (or laboratory angle). The net negative transverse momentum is the signal for collective flow, a directed momentum transfer which can only be understood in terms of the nuclear equation-of-state. These two plots show the magnitude of the flow for two different trigger conditions. 


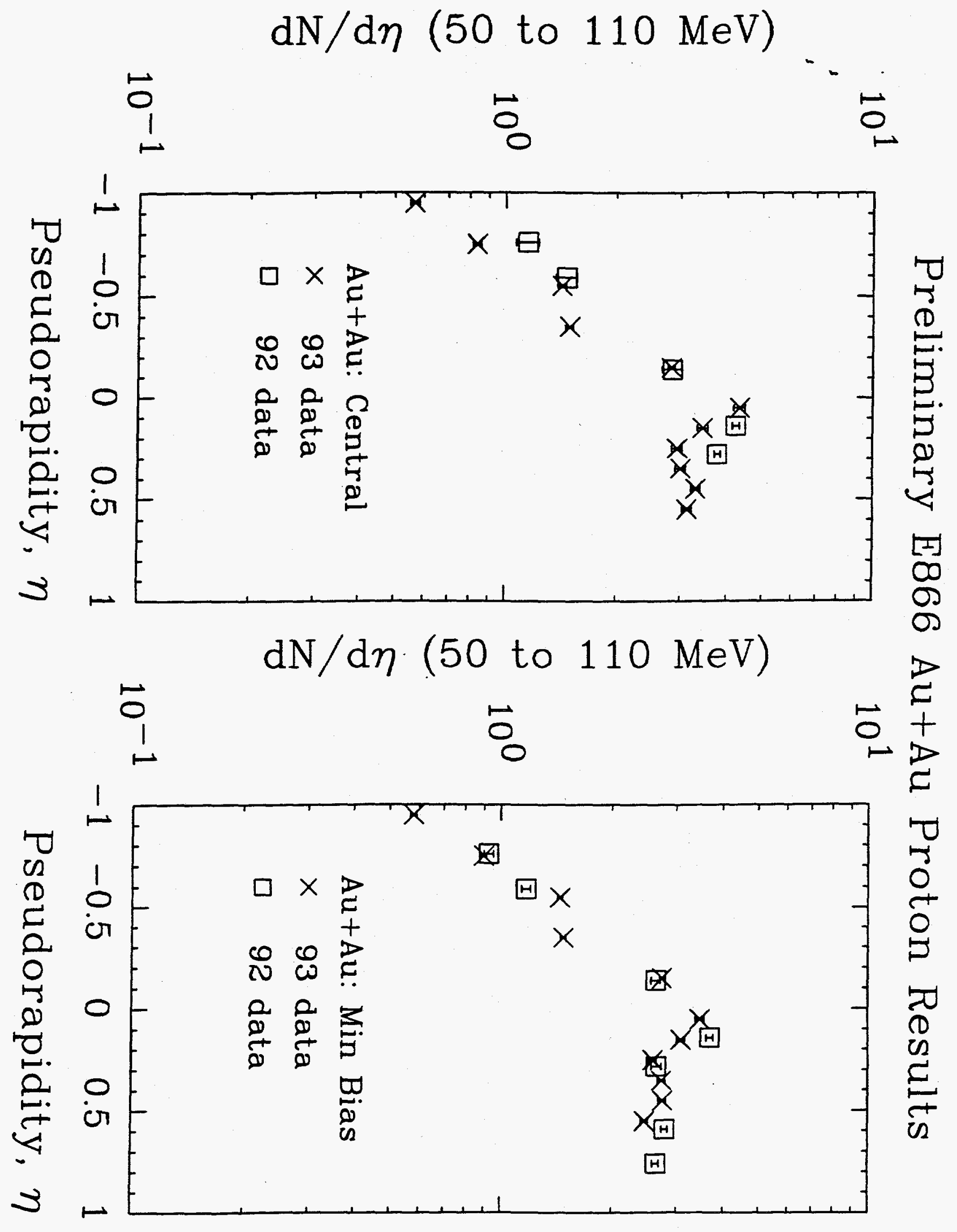

Figure 1 
Hodo Slat 14, Run 14766
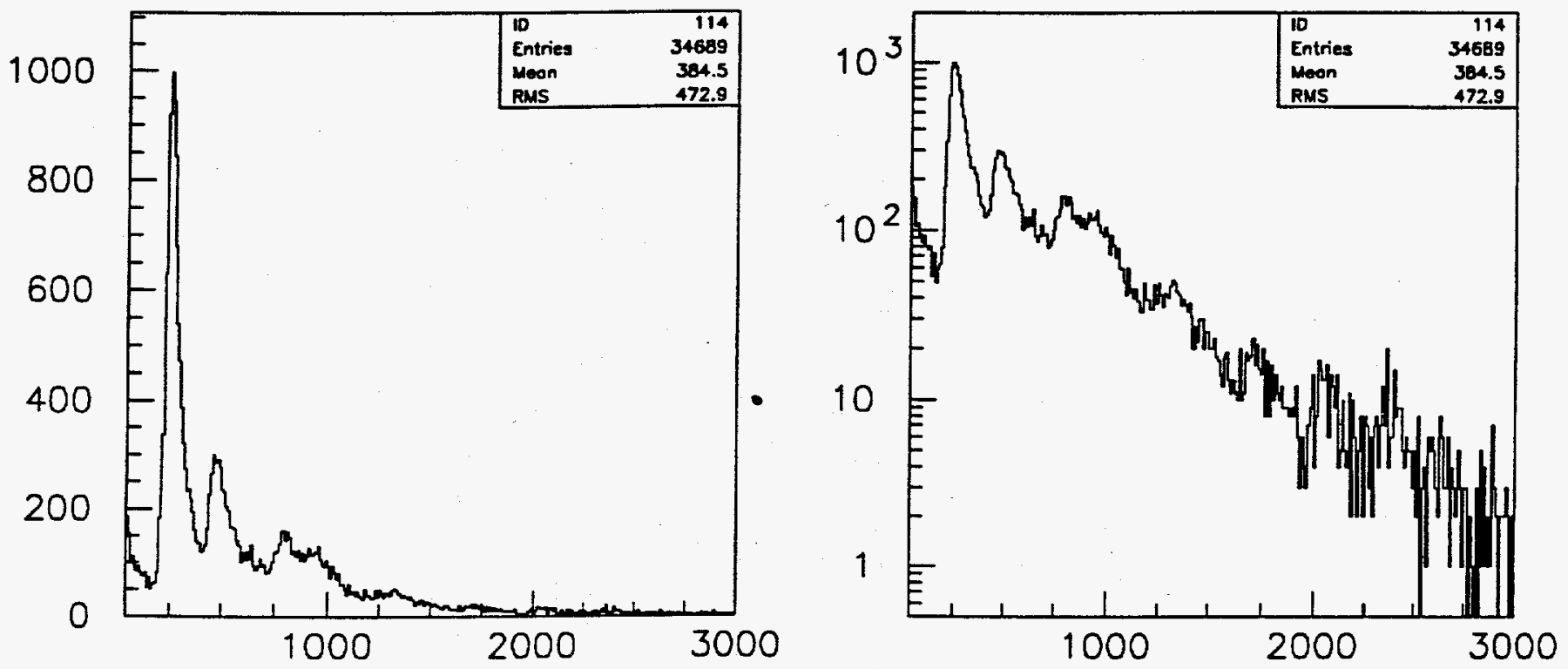

Geo Mean, X14
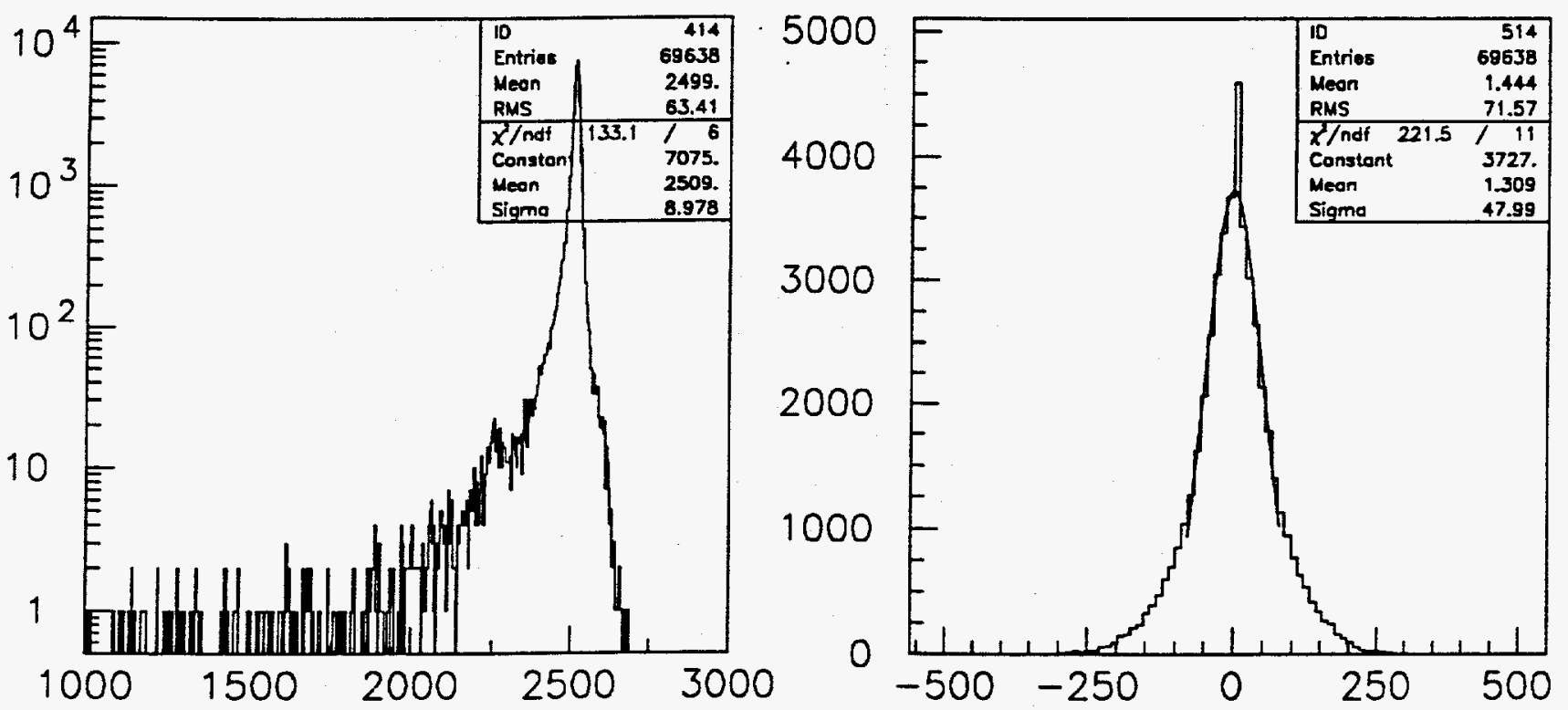

Time sum for $\times 14$

Time dif for $\times 14$

Figure 2 


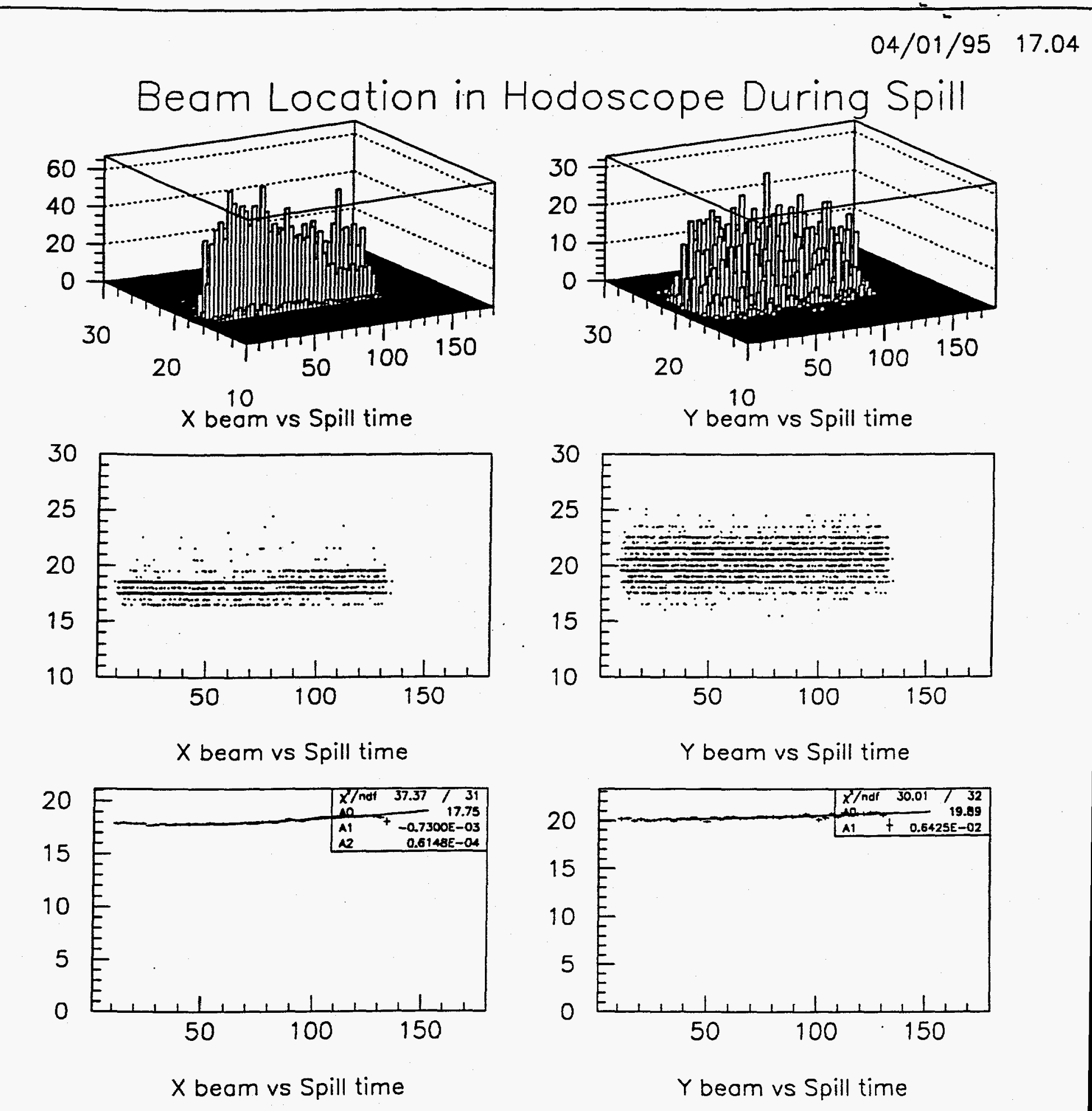

Figure 3 
Preliminary E866 Au+Au

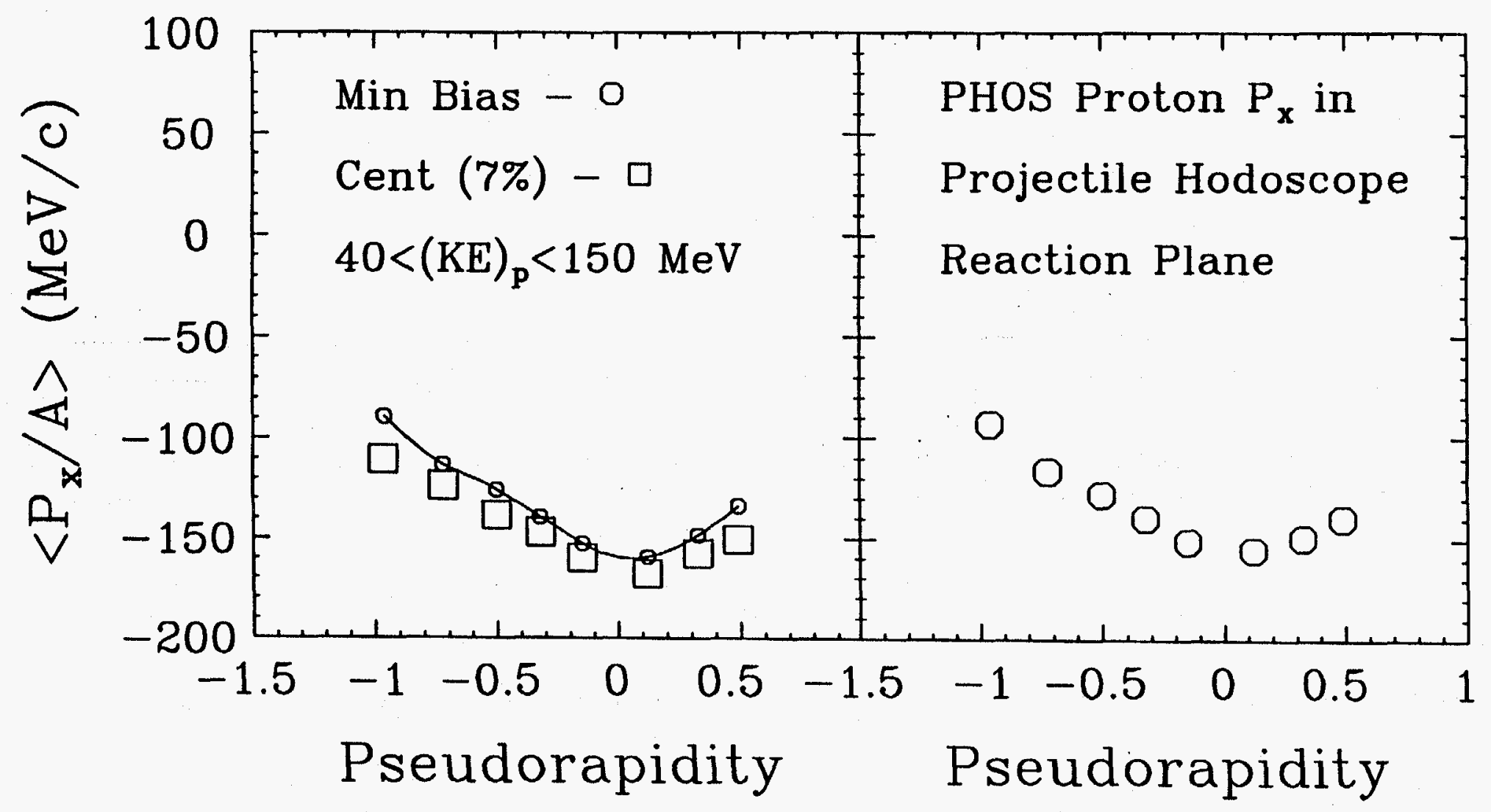

